rev Psi

Revista de Psicología (UNLP)

https://revistas.unlp.edu.ar/revpsi

\title{
¿Qué intervenciones pueden tener los analistas en el lazo social?
}

\author{
Marcelo Ale ${ }^{1}$ \\ Correspondencia \\ marcelorale@yahoo.com.ar \\ Filiaciones institucionales \\ ${ }^{1}$ Participante de las actividades de la Escuela de \\ Orientación Lacaniana (EOL, Argentina)
}

\section{Resumen}

Si a los analistas nos interesa la cultura en la que vivimos es porque esta incide sobre la práctica analítica. La clínica no es impermeable al estado contemporáneo de la cultura que desembarca con sus discursos dominantes en los divanes. Las teorías del malestar dependen de la época en la que nos toca vivir. En psicoanálisis no hay modo de anoticiarnos de ese malestar sino es por su portavoz, el síntoma, que siempre ha tenido una envoltura formal, un modo de presentación que depende del Otro al cual se refiere. Las épocas, definidas por el predominio de los discursos imperantes, varían. Es diferente la época victoriana en la que Freud funda el psicoanálisis que la actual: en aquella se tendía a la represión de la satisfacción sexual, en esta a su promoción. Si las épocas varían, el psicoanálisis está obligado a reconsiderar su "intervención en lo social," instalándose esta como un tema de debate permanente.

\section{Palabras clave}

síntomas | discursos | cultura | psicoanálisis | interpretación

\section{Proceso editorial}

Recibido

1 dic. 2020

Aceptado

6 jun. 2021

1 ra decisión

23 mar. 2021

Publicado

9 jun. 2021

\section{Editores}

Stella López (Facultad de Psicología UNLP, Argentina) y Nicolás Alessandroni (Facultad de Psicología UAM, España).
ISSN

2422-572X

Licencia

Licencia de Cultura Libre CC-BY 4.0

(Compartir - Adaptar - Atribuir)

Entidad editora

RevPsi es una publicación de la

Facultad de Psicología (Universidad

Nacional de La Plata, Argentina) 


\section{Que intervenções podem ter os analistas no vínculo social?}

\section{Resumo}

Se nós analistas estamos interessados na cultura em que vivemos, é porque ela tem um impacto sobre a prática analítica. A clínica não é impermeável ao estado contemporâneo da cultura que desembarca com seus discursos dominantes sobre os sofás. As teorias do desconforto dependem da época em que vivemos. Na psicanálise, não há como estar consciente deste mal-estar, a não ser através de seu porta-voz, o sintoma, que sempre teve um envelope formal, um modo de apresentação que depende do Outro a quem se refere. As épocas, definidas pela predominância dos discursos predominantes, variam. A época vitoriana na qual Freud fundou a psicanálise é diferente da atual: na primeira havia uma tendência a reprimir a satisfação sexual, na segunda a promovê-la. Se as épocas variam, a psicanálise é obrigada a reconsiderar sua "intervenção no social", estabelecendo-se como um tema de debate permanente.

\section{Palavras-chave}

sintomas | discursos | cultura | psicanálise | interpretação

\section{What interventions can analysts have in the social bond?}

\section{Abstract}

If we analysts are interested in the culture in which we live, it is because it has an impact on analytic practice. The clinic is not impervious to the contemporary state of the culture that disembarks with its dominant discourses on the couches. The theories of malaise depend on the era in which we live. In psychoanalysis, there is no way of being aware of this malaise except through its spokesperson, the symptom, which has always had a formal envelope, a mode of presentation that depends on the Other to whom it refers. The epochs, defined by the predominance of the prevailing discourses, vary. The Victorian era in which Freud founded psychoanalysis is different from the present one: in the former, the tendency was towards the repression of sexual satisfaction, in the latter towards its promotion. If the times change, psychoanalysis is obliged to reconsider its "intervention in the social," and this becomes a subject of permanent debate.

\section{Keywords}

symptoms | discourses | culture | psychoanalysis | interpretation 


\section{¿Una paradoja a develar?}

Si bien la interpretación analítica apunta a desconectar al significante del significado, esto es a producir nuevos efectos de significación, también se dirige a la relación del sujeto con su modo de gozar, es decir, con la satisfacción pulsional.

Es esta segunda acepción la que puede producir una verdadera mutación subjetiva, ya que la primera si bien provoca nuevos efectos de significación en los registros simbólico/imaginario, no necesariamente impacta en la relación del sujeto con la satisfacción pulsional real.

Esta segunda acepción está acentuada en la fundamentación de este dossier de Revista de Psicología UNLP, en el párrafo escrito por Stella López en el que se lee que: "La operación analítica se sitúa a nivel del goce. Además de esta advertencia de Freud, se rescata otra según la cual previene a los analistas de no volverse fanático de la salud cuando el sujeto ha conquistado con su manera de gozar un lugar en el lazo social". Quedan delimitadas así "la ganancia primaria y secundaria del síntoma, su aspecto social, compuesto más por su satisfacción que por su sentido. Es constatable que existe una tensión entre el discurso analítico apoyado en la singularidad del síntoma y el discurso del amo". Continua afirmando: "la psicología de masas freudiana sigue vigente, cómo cambian los regímenes identificatorios, su organización, qué ocurre con la función del líder, cómo se manifiesta en esta época la pulsión de muerte en los lazos identificatorios, cómo situar las nuevas segregaciones, entre otros temas posibles".

Para comenzar a desarrollar el tema, algunas preguntas: ¿Cómo se manifiesta hoy esa psicología de masas y el "malestar en la cultura”? ¿Como definimos el lazo social? ¿Es diferente de "lo social"? ¿Qué lo ordena? ¿La referencia a un significante amo? ¿La identificación a un modo de gozar que haga comunidad?

Entiendo que las respuestas que podamos dar a estos interrogantes definirán el modo de intervención que los analistas tengamos en el lazo social.

Con aquellas dos acepciones de la interpretación, tenemos así presentes el sentido y el goce en el síntoma, ese goce que a partir de la última cita, lejos de ser algo de lo que habría que "operar" al sujeto, extirpárselo, también opera como lo que "ordena" su manera de vivir = gozar en el lazo social.

Tenemos el goce en el síntoma, que es lo que pretende transformar la interpretación, que al mismo tiempo funciona "ordenando" a cada uno, la manera de gozar en el lazo social. ¿Qué consecuencias podrá generar la paradoja de transformar lo que funciona? ¿Paradoja a develar?

\section{¿Sobre qué forma de " lazo social" intervenimos?}

Si planteamos una intervención posible de los psicoanálistas en "el lazo social", es pertinente aclarar previamente a qué acepción de "lo social" nos referimos, es decir, ¿cómo consideramos ese campo sobre el que el psicoanálisis podría intervenir? 
La definición de "lazo social" que da Lacan es diferente de la que podemos encontrar en las acepciones clásicas en el campo de la sociología y las psicologías ya que, como sí sucede en estas, no se refiere a las relaciones entre los individuos en sociedad, sino a las que mantiene el sujeto con los discursos: el lazo social se refiere a la relación del sujeto con los discursos, no a la de un individuo con otro. Primera cuestión a esclarecer para ir delimitando el campo de intervención: ¿que entiende Lacan por "lo social" sobre lo que se podría intervenir?

Si el lazo social se apoya en la relación del sujeto con los discursos, podríamos revisar la teoría lacaniana de los discursos como formas del lazo social. Teorías de los discursos que desarrolla en los Seminarios 16 De un Otro al otro y en el 17 El reverso del psicoanálisis, cuando se refiere a los discursos del amo, histérico, universitario y analítico, como así también al que se refirió pocas veces bajo el nombre de discurso capitalista (hoy podríamos decir "neoliberal") que se trata de la promoción de la conjunción del sujeto con su objeto, vía el empuje al consumo en cuyo horizonte espera la promesa de felicidad. La estructura del discurso capitalista está apoyada en la conjunción del sujeto con su objeto de satisfacción, el discurso neoliberal lo promueve, alienta y presenta como horizonte al consumidor.

Recojo una referencia a este último discurso, planteada en el año 1972 en la llamada Conferencia de Milán ${ }^{1}$ donde sostiene que: "Apenas he dicho lo que es un discurso. El discurso ¿qué es? Es lo que, en el orden... en la disposición de lo que puede producirse por la existencia del lenguaje, tiene la función de lazo social".

Respecto del discurso capitalista, afirma que es el sustituto del discurso amo: “ (...) es un discurso locamente astuto y en crisis, que está destinado a estallar, que es a todas luces insostenible. Como dijimos oportunamente lo que los diferencia es que, entre ambos, sólo hay una pequeña inversión entre el S1 y el \$, el sujeto. Como resultante obtenemos un "eso marcha", marcha maravillosamente, marcha tan bien que se consuma y, en el mismo acto, se consume a sí mismo".

En otro texto, titulado Hablo a las paredes ${ }^{2}$ dirá que el discurso del capitalismo es lo mismo que el del amo, sólo que está hecho mejor en tanto y en cuanto funciona bien y nos embauca más, “itanto que no nos damos ni cuenta!”.

Cabe preguntarnos ¿qué es lo que funciona y por qué nos embauca?

El capitalista es un discurso en el que el sujeto está en una relación de conjunción con su objeto (a partir de esa inversión que señalara entre el S1 y el \$ en el lugar del agente), relación en la que no hay resto, no hay pérdida. El objeto está llamado a suturar la división subjetiva, siendo así la fórmula del fantasma realizada en su máxima expresión y encarnada fenomenológicamente en la promesa de felicidad a la que apunta y sostiene.

El capitalista devenido en neoliberal, entonces, es un discurso que construye una subjetividad en la que la castración está apartada, esquivada, ocultada, velada. Así, promesa del discurso neoliberal y construcción de la subjetividad que vela la castración, se conectan, siendo sobre este "lazo social" del sujeto con este discurso, 
sobre el que la intervención analítica debe desembarcar.

Este concepto de "lazo social" construido a partir de la teoría de los discursos, es empleado por Lacan en lugar del de "sociedad", a la vez que advertimos que hay tantos lazos sociales como discursos. Siendo el capitalista, hoy neoliberal, el blanco de la intervención.

\section{¿Qué ordenan cultura y pulsión en el lazo social?}

Es sabido que la cultura de hoy no es la misma que la de Freud cuando escribió su texto El Malestar en la cultura -texto anticipado veinte años antes en su otro artículo La moral sexual cultural y la nerviosidad moderna-, por lo tanto las tesis sobre las causas de ese malestar tampoco son iguales.

Si a los analistas nos interesa el estado de la cultura en la que vivimos, es porque esta incide sobre la práctica analítica, de manera que la clínica no es impermeable al estado contemporáneo de la cultura que desembarca con sus discursos dominantes en los consultorios.

El malestar actual no se plantea según el modelo freudiano de la represión, porque este es un momento de la historia que, lejos de prohibir el goce, lo exige. Es una época en la que predomina la doctrina del capitalismo llamado "mercado global", en la que la pretendida universalidad a la que aspira la globalización, impide paradójicamente, establecer reglas válidas para todos.

Es el campo de la cultura el espacio donde se inventan las formas de gozar, es decir, de satisfacer la pulsión, por lo tanto si la cultura cambia, esas formas de satisfacción son móviles, variables. De este modo, esta se erige como el Otro de la pulsión que contiene sus objetos.

Jacques Lacan en su Seminario R.S.I., consideraba que el origen de la noción de síntoma no hay que ir a buscarlo hasta Hipócrates, sino en Karl Marx; más precisamente en lo que él consideraba que derivaba de la relación entre el capitalismo y el tiempo del feudalismo, cuando afirmaba que el capitalismo tenía efectos que se expresaban en las masas sociales como malestares que irrumpen al modo de un signo que expresa lo que no funciona.

Es preciso subrayar, como lo he destacado, que no es del capitalismo como teoría y práctica política de la sociedad de lo que se trata sino del "discurso capitalista", hoy neoliberal, en el sentido en el que propone una nueva forma de la subjetividad que produce sus consecuencias en los sujetos que lo consumen. Ya que en esta economía actual, lo que se produce y consume no son solo mercancías sino discursos que, finalmente, terminan cobrando valor de mercancía.

Para Marx, son síntomas una serie de hechos históricos que surgen como indicio de lo que no marcha para la propuesta del discurso del amo: desempleo, crisis, manifestaciones, huelgas que van surgiendo en el pasaje de una estructura económica a otra. Hay una sociedad que tiende a un modo de funcionamiento 
propuesto por el discurso amo, y manifestaciones disruptivas que indican que eso no marcha. Así el síntoma, tal como afirmaba Lacan en el año 1968, es aquello que se pone en cruz para impedir que las cosas anden de manera satisfactoria para el amo.

Una teoría del malestar no es sin su dependencia de la época en la que nos toca vivir. En psicoanálisis no hay modo de anoticiarnos de ese malestar sino es por su portavoz, el síntoma, que siempre ha tenido una envoltura formal, un modo de presentación que depende del Otro al cual se refiere. Se trata de que hable en el lenguaje del Otro al cual se dirige como mensaje y enigma. Debe pasar de eso que puede resultar impreciso, molesto e inquietante, a un lenguaje que el Otro de turno pueda descifrar. Por lo tanto, no hay síntoma como portavoz del malestar que no apele a los significantes de la cultura del momento que alimentan su texto: esto es su envoltura formal.

Freud designaba malestar a la expresión subjetiva del retorno de aquello que el sujeto debió ceder para entrar en el campo del Otro. Ser parte del rebaño le cuesta ceder sobre su deseo a las proscripciones y prescripciones de la cultura de su tiempo.

¿Por qué a los analistas nos interesan la cultura, los fenómenos sociales, la psicología colectiva, las formaciones de masa, su actualidad y sus variaciones? ¿Qué nos lleva por la senda del analista ciudadano a salir a la Polis y debatir sobre los temas de actualidad en el campo social?

Freud llamó malestar a la expresión subjetiva del retorno de aquello que el sujeto debió ceder para entrar en el campo del Otro y ser parte de "lo social", y definió a la cultura en una de sus acepciones, como ese aparato para regular ese real ingobernable que es la pulsión -demostrando así que la operación analítica se sitúa a nivel de la relación del sujeto con el goce- y por otro lado como el lugar en el que se crean las formas de satisfacer esa pulsión.

Entonces se impone nuevamente la pregunta: ¿cómo intervenir en lo social para incidir sobre esa subjetividad?

\section{¿Cómo responder a la promesa del bienestar que pregona el discurso neoliberal?}

Según planteaba Eric Laurent en diciembre de 2007 en Buenos Aires ${ }^{3}$, esta es una época en donde se pone en juego un pasaje de la biopolítica del estado, que directa e indirectamente castiga a los ciudadanos, al auto castigo. Se introduce así un más allá de la victimización Foucaultiana, en términos que ese castigo ya no está del lado de las organizaciones gubernamentales, sino del lado mismo del sujeto como enemigo propio. Es decir que los síntomas actuales, ya no son las marcas que las víctimas reciben del otro, sino los distintos modos que el sujeto encuentra de poner un juego el deseo más decidido del neurótico que es, tal como le gustaba decir a Freud, el de gozar de su castración.

Es la época del empuje a gozar sobre el que actúa subterráneamente el principio de 
felicidad de Jeremy Bentham. Este principio alimenta el proyecto del liberalismo económico y político y se actualiza en las promesas de bienestar para todos. Es la promesa de que todo es posible alcanzar y que nada quedará por fuera de la satisfacción, discurso que paradójicamente ordena gozar en el ejercicio de la libertad que promete y que causa, o modos de satisfacción que cierran el inconsciente, o síntomas como demandas sociales.

Podríamos preguntarnos si los interrogantes sobre los modos de intervención en "lo social" no deberían sustentarse en las siguientes preguntas: ¿Cómo incluir, en la dictadura del discurso que graba la orden de "gozar sin límites", la democracia de la castración como corte entre el sujeto y el objeto para que este pueda decir "no" y acceder a un saber sobre la verdad que lo determina?

Destacó Éric Laurent en esa misma conferencia que las encuestas realizadas en EE.UU. desde hace 40 años y en Europa desde hace 20 aproximadamente demostraron que los índices de felicidad no mejoraron a pesar de las políticas que pretendían promoverlos y que en Inglaterra, luego de la instalación de 12000 cámaras vigilantes como paradigma del panóptico, ellos comenzaron a ser sustituidos por un sentimiento de desconfianza. De este modo, se establece una nueva categoría: la de "los depresivos resistentes" a la terapéutica de la felicidad. Este principio que promete el bienestar para todos introduce un imperativo: "Todo lo posible es obligatorio".

\section{¿Como se construye la subjetividad que "ordena" el lazo social?}

¿De qué modo se construye la subjetividad en una época? Un modo de concebirla es a partir de la estructura de la sugestión. y su extensión a lo social.

Ernesto Laclau en los capítulos 2 y 3 de su libro La razón populista, titulados "Le bon: sugestión y representaciones distorsionadas", y "Sugestión, imitación, identificación” sigue el camino de la referencia que Freud, para teorizar sobre la psicología de las masas, toma del libro de Le bon titulado Psicología de las multitudes. Es en ese libro donde el autor se refiere a la clave de la influencia que ejercen las palabras en la formación de una masa, clave que Laclau resume en estos términos: "Según Le bon existen tres recursos: la afirmación, la repetición y el contagio". Cita a Le bon: "La afirmación pura y simple libre de todo razonamiento y de toda prueba, es uno de los medios más seguros para introducir una idea en la mente de una masa. Cuanto más concisa es la afirmación, mayor es su influencia". Continúa: "en cuanto a la repetición, su poder se debe al hecho de que la afirmación repetida se fija, en el largo plazo, en aquellas regiones profundas de nuestro yo inconsciente en la cual se forjan las motivaciones de nuestras acciones. Al pasar cierto tiempo, olvidamos quien es el autor de la afirmación repetida y terminamos por creer en ella". Finalmente, respecto del contagio afirma que es un fenómeno muy natural que se observa también en animales cuando están juntos en cantidad.

En Psicología de las masas y análisis del yo, Freud afirmaba: “(...) el vínculo hipnótico 
es una formación de masa de dos" (1992, p. 108) y debe su efecto al poder sugestivo de las palabras. Esta sugestión hipnótica, además de ser un fenómeno específico de los estados patológicos individuales como se creyó en su inicio, es considerada además un efecto de la palabra en los comportamientos de las masas: se trata, en este caso, de una hipnosis social. Así, afirma Laclau que "La psicología de las masas ha tocado algunos aspectos de crucial importancia en la construcción de las identidades políticas, y sociales (...) El predominio de lo emotivo por sobre lo racional, la sugestibilidad, y la identificación con los líderes, constituyen rasgos reales del comportamiento colectivo".

Sugestión hipnótica, formación de masa y construcción de subjetividad, se enlazan así en la sobre determinación del "lazo social" que mantiene el sujeto con el discurso neoliberal.

En su libro Horizontes neoliberales en la subjetividad, Jorge Alemán plantea que la subjetividad construida hoy por el discurso neoliberal, se presenta con el disfraz del "hombre nuevo como gerente de sí, se configura según un paradigma empresarial competitivo y gerencial de la propia existencia y se expresa, por ejemplo, en los fenómenos de la autoayuda".

Por otro lado, también se refiere a dos estudiosos del tema, Christian Laval y Pierre Dardot, quienes recuerdan a Margaret Thatcher cuando decía, respecto de los objetivos del neoliberalismo, que "la economía es el método pero el objetivo es el alma”, es decir que el propósito es fabricar subjetividades. Es interesante subrayar esta definición porque abre el debate respeto de la intencionalidad del discurso, ya que habitualmente se tiende a considerar que apunta a la economía.

Ese discurso está basado en la exigencia de rendimiento y goce que retorna en los fenómenos de la depresión, el consumo de fármacos, la asunción como problema personal de lo que es estructural, la responsabilidad desmedida, etc. El imperativo de felicidad y su contracara, la depresión en la época, es una de sus consecuencias, termina afirmando.

En el marco de los efectos discursivos en el "oyente", cabe preguntarnos si esa exigencia de imposible realización, lejos de permitir arribar a la felicidad prometida, no introduce un nuevo malestar en la cultura.

\section{Conclusiones...abiertas}

La pregunta que a mi entender hay que tratar de responder, porque contiene el interrogante por el futuro del psicoanálisis en el mundo y por lo tanto de su posibilidad de incidir en el lazo social es: ¿Hasta dónde puede subsistir el psicoanálisis bajo la constelación discursiva del discurso neoliberal que, como nueva producción de la subjetividad, ofrece una relación de conjunción entre el sujeto y su objeto opuesta a la que requiere la realización de la experiencia analítica? 
La problemática de la coexistencia de ambos discursos se establece porque el discurso neoliberal como nueva realización de la subjetividad, propone y ofrece un tipo de relación entre el sujeto y el goce -que es opuesto al que requiere la condición de posibilidad de la experiencia analítica -ya que se trata de una relación de conjunción en la medida que el objeto técnico como mercancía que prolifera gracias al ciencia, viene a suturar la división subjetiva. En términos psicoanalíticos, diríamos que lo que establece es la consolidación del fantasma.

Sabemos por otro lado, que si no hay ruptura de esa comunión, sino de diluye ese consentimiento, sino hay desestabilización por tanto del fantasma, no puede haber inicio del la experiencia analítica. Al respecto afirma Jorge Alemán en su libro La experiencia del fin-psicoanálisis y metafísica: “...el capitalismo asegura un nuevo tipo de cierre del inconsciente propiciado por un fantasma que ha incluido al fin al objeto técnico en la economía libidinal".

¿Cómo salir del discurso neoliberal, entonces? Si el psicoanálisis va a seguir existiendo si y solo si persiste el síntoma que supone la discordia y no el consentimiento del sujeto con el goce, se impone preguntarnos: ¿qué es lo que puede hacer prevalecer en la decisión subjetiva de cada uno, que se adopte por una u otra relación al goce?

Concluyo este punto con algunos interrogantes del autor en este último libro, que invitan a reformular y revisar la presencia del psicoanálisis en el mundo, y por lo tanto la posibilidad de incidir en el lazo social: “¿Qué quiere decir Lacan cuando habla de salida del capitalismo y sin embargo no opta por el socialismo? ¿A qué dimensión de lo subjetivo apunta? ¿Cómo habita, como tiene lugar el psicoanálisis en la época de la ciencia? ¿Qué quiere decir cuando invita a darle el deber que le corresponde en el mundo?"

Arriesgo una respuesta del mismo autor, en otro de sus libros titulado Cuestiones antifilosóficas en J. Lacan, cuando en el marco del planteo que el discurso analítico lleva el saber al lugar de la verdad, afirma que se trata de: "desentrañar la relación del sujeto con el plus de goce implícito en el valor del objeto técnico y transmitir el saber de esa operación antes que la humanidad, como decía Lacan en Roma, logre curarse del psicoanálisis".

Lacan en La Tercera, sobre el porvenir del primero en la segunda, afirmó que el psicoanálisis permanecerá siendo síntoma de la cultura en tanto pueda agujerear su campo de significados, y que además tiene que introducir algo que no sea captado en ese campo semántico que enmarca la producción de la subjetividad; esto último dará la condición de su subsistencia.

La producción de la subjetividad como efecto el discurso mediático, implica la construcción de yoes que se identifican entre sí en un efecto de masa que al homogeneizarlos, reduce sus diferencias. La subjetividad es una construcción social que formatea al yo y eclipsa al sujeto del inconsciente. En cambio, ese sujeto como efecto del lenguaje, pone en juego lo que la masificación no capta y conserva su ex- 
sistencia si lo que lo singulariza no es asimilado en el campo de la cultura. De no ser así, el discurso lo eclipsa

Se trata, por el lado de la subjetividad, de una tendencia a la homogenización que conlleva el borramiento del sujeto como resultado de la eficacia del discurso del neoliberalismo, y por el lado del sujeto, de la creación del síntoma particular como lo más propio de cada uno que es introducido como un cuerpo extraño en aquella. El sujeto con la subjetividad construida, debe mantener la misma relación de extraterritorialidad que el síntoma con el yo, debe ser en tal sentido, egodistónico con la cultura, si se enquista en ella y se hace culturasintónico, desaparece.

La hipótesis del sujeto como efecto del lenguaje que introduce las diferencias singulares, supone un límite al avance del totalitarismo discursivo que alimenta la homogeneidad en la creación de la subjetividad de masas.

El eclipse del sujeto, se sentencia en la realización del discurso capitalista, su subsistencia en cambio coexiste en el discurso del inconsciente en tanto, en su piso inferior, se manifiesta la disyunción del sujeto con el objeto, ( $\$ / / a)$ ya que no se conectan, queda un resto inasimilable que no es captado en la masificación y demuestra que la subjetividad producida no lo eclipsa.

La intervención en "lo social" debe apuntar a que ese resto inasimilable deba surgir y subsistir. Apunta a "deshipnotizar", a apartar al sujeto del eclipse en la masificación y así conservar su singularidad.

¿En esa intervención en "lo social", se trata solamente, como la ocasión, de estar atentos al salto del león de lo inasimilable para que las huellas del sujeto no sean borradas y su desaparición no acontezca?

\section{Notas}

${ }^{1}$ Del discurso psicoanalítico, conferencia en Milán del 12 de mayo de 1972. Traducción: Olga Mabel Máter. https://elpsicoanalistalector.blogspot.com.ar ${ }^{2}$ Lacan, J. (2012). Hablo a las paredes. Paidós.

${ }^{3}$ Variaciones de la cura analítica, hoy. La relación entre el efecto terapéutico y su más allá. Conferencia de apertura a la XVI Jornadas Anuales de la Escuela de la Orientación Lacaniana. 1 y 2 de diciembre de 2007, Marriott Plaza Hotel, Buenos Aires (Argentina). 


\section{Referencias}

Alemán, J. (1992). Cuestiones antifilosóficas en J. Lacan. Editorial Atuel.

Alemán, J. (1996). La experiencia del fin-psicoanálisis y metafísica. Miguel Gómez Peña Ediciones.

Alemán, J. (2016). Horizontes neoliberales en la subjetividad. Grama.

Freud, S. (1987). La moral sexual cultural y la nerviosidad moderna. En sus Obras completas. Tomo IX. Amorrortu.

Freud, S. (1992). Psicología de las masas y análisis de yo. En sus Obras completas. Tomo XVIII. Amorrortu.
Freud, S. (1987). El malestar en la cultura. En sus Obras completas. Tomo XX. Amorrortu.

Laclau, E. (2005). La razón populista. FCE.

Lacan, J. (1991). La tercera. En Intervenciones y textos 2. Manantial.

Lacan, J. (2008). El seminario. Libro 16. De un Otro al otro. Paidós.

Lacan, J. (1992). El seminario. Libro 17. El reverso del psicoanálisis. Paidós. 